\title{
Numerical Simulation Analysis of a Mathematical Model of Circadian Pacemaker Neurons
}

\author{
Takaaki Shirahata \\ Kagawa School of Pharmaceutical Sciences, Tokushima Bunri University, Sanuki, Japan \\ Email: tshi@kph.bunri-u.ac.jp
}

Received 16 June 2015; accepted 7 July 2015; published 10 July 2015

Copyright (C) 2015 by author and Scientific Research Publishing Inc. This work is licensed under the Creative Commons Attribution International License (CC BY). http://creativecommons.org/licenses/by/4.0/ c) (i) Open Access

\begin{abstract}
Sim and Forger have proposed a mathematical model of circadian pacemaker neurons in the suprachiasmatic nucleus (SCN). This model, which has been formulated on the Hodgkin-Huxley model, is described by a system of nonlinear ordinary differential equations. An important feature of the SCN neurons observed in electrophysiological recording is spontaneous repetitive spiking, which is reproduced using this model. In the present study, numerical simulation analysis of this model was performed to evaluate variations in two system parameters of this model: the maximal conductance of calcium current $\left(g_{C a}\right)$ and the maximal conductance of sodium current $\left(g_{N a}\right)$. Simulation results revealed the spontaneous repetitive spiking states of the model in the $\left(g_{C a}, g_{N a}\right)$-parameter space.
\end{abstract}

\section{Keywords}

Mathematical Model, Numerical Simulation, Neuron, Spiking

\section{Introduction}

Repetitive spiking activity is an important feature of excitable cells such as neurons and muscle cells. In the field of applied mathematics, this activity can be extensively analyzed using various mathematical models, which are described by a system of nonlinear ordinary differential equations (ODEs). One example is the Hodgkin-Huxley model, which can generate repetitive spiking because of an interaction between the sodium conductance and the potassium conductance (page 25 in [1]). In this model, the sodium conductance plays an excitatory role, whereas the potassium conductance plays an inhibitory role. The other example is the Morris-Lecar model, which can also generate repetitive spiking because of an interaction between the calcium conductance and the potassium 
conductance (page 49 in [1]). In this model, the calcium conductance and not the sodium conductance plays an excitatory role.

Another type of mathematical model of the repetitive spiking activity is the one in which the repetitive spiking is generated by an interaction among sodium, calcium, and potassium conductances. One example is a mathematical model of circadian pacemaker neurons in the suprachiasmatic nucleus (SCN) [2], which is described by a system of nonlinear ODEs (see the Materials and Methods in [2] as well as the Materials and Methods of this manuscript). Considering the Hodgkin-Huxley and Morris-Lecar models described above, it is assumed that both the sodium and calcium conductances play excitatory roles in the circadian pacemaker neuron model. However, the difference in the contribution to the repetitive spiking between the sodium and calcium conductances was not clarified in detail in a previous study [2]. In particular, the influence of variations in these two conductance values on the repetitive spiking was not studied in detail in that study [2]. Taking into consideration that it is not only interesting but also very important to analyze the ionic conductance in detail (page 26 in [3]), it is necessary to investigate the influence of variations in these two conductance values and reveal the difference between these two conductances. Therefore, in the present study, we focused on two system parameters of the circadian pacemaker neuron model, namely the maximal conductance of calcium current $\left(g_{C a}\right)$ and the maximal conductance of sodium current $\left(g_{N a}\right)$, and we performed numerical simulation analysis.

\section{Materials and Methods}

A mathematical model of the circadian pacemaker neuron, which has been used in the present study, was a model developed by Sim and Forger [2]. This model is described by a system of nonlinear ODEs and contains six state variables: a membrane potential of the circadian pacemaker neuron $[V(\mathrm{mV})]$ and five gating variables of ionic currents ( $m, h, n, r$, and $f$ ). The time evolution of the six state variables is described as follows:

$$
\begin{aligned}
& C \frac{\mathrm{d} V}{\mathrm{~d} t}=I_{a p p}-I_{N a}(V, m, h)-I_{K}(V, n)-I_{L}(V)-I_{C a}(V, r, f) \\
& \frac{\mathrm{d} X}{\mathrm{~d} t}=\frac{1}{\tau_{X}(V)}\left(X_{\infty}(V)-X\right) \quad(X=m, h, n, r, f)
\end{aligned}
$$

where $C$ is membrane capacitance (5.7 pF); $I_{N a}(V, m, h), I_{K}(V, n), I_{L}(V)$, and $I_{C a}(V, r, f)$ are a sodium current, a potassium current, a leak current, and a calcium current, respectively, which are defined in Equations (3)-(6) below, in this order; $\tau_{X}(V)$ (ms) and $X_{\infty}(V)$ are time constants of activation/inactivation and steady-state activation/inactivation functions, respectively, which are defined in Equations (7)-(16) below, in this order; and $I_{a p p}$ is applied current.

$$
\begin{aligned}
& I_{N a}(V, m, h)=g_{N a} m^{3} h\left(V-E_{N a}\right) \\
& I_{K}(V, n)=g_{K} n^{4}\left(V-E_{K}\right) \\
& I_{L}(V)=g_{L}\left(V-E_{L}\right) \\
& I_{C a}(V, r, f)=g_{C a} r f\left(V-E_{C a}\right)
\end{aligned}
$$

where $g_{N a}, g_{K}, g_{L}$, and $g_{C a}$ are the maximal conductances of a sodium current (the $g_{N a}$ value was varied in the present study; the default value was $229 \mathrm{nS})$, a potassium current (14 nS), a leak current (1/11 nS), and a calcium current (the $g_{C a}$ value was varied in the present study; the default value was $65 \mathrm{nS}$ ), respectively; $E_{N a}, E_{K}$, $E_{L}$, and $E_{C a}$ are the reversal potentials of a sodium current $(45 \mathrm{mV})$, a potassium current ( $\left.-97 \mathrm{mV}\right)$, a leak current $(-29 \mathrm{mV})$, and a calcium current $(61 \mathrm{mV})$, respectively.

$$
\begin{aligned}
& \tau_{m}(V)=\mathrm{e}^{-(V+286) / 160} \\
& \tau_{h}(V)=0.51+\mathrm{e}^{-(V+26.6) / 7.1} \\
& \tau_{n}(V)=\mathrm{e}^{-(V-67) / 68}
\end{aligned}
$$




$$
\begin{aligned}
& \tau_{r}=3.1 \\
& \tau_{f}(V)=\mathrm{e}^{-(V-444) / 220} \\
& m_{\infty}(V)=\frac{1}{1+\mathrm{e}^{-(V+35.2) / 7.9}} \\
& h_{\infty}(V)=\frac{1}{1+\mathrm{e}^{(V+62) / 5.5}} \\
& n_{\infty}(V)=\frac{1}{\left(1+\mathrm{e}^{-(V-14) / 17}\right)^{0.25}} \\
& r_{\infty}(V)=\frac{1}{1+\mathrm{e}^{-(V+25) / 7.5}} \\
& f_{\infty}(V)=\frac{1}{1+\mathrm{e}^{(V+260) / 65}}
\end{aligned}
$$

Detailed explanations of the model equations have been described previously [2].

The free and open source software Scilab (http://www.scilab.org/) was used to numerically solve ODEs (initial conditions: $V=-80 \mathrm{mV}, m=0.34, h=0.045, n=0.54, r=0.01, f=0.04)$.

\section{Results}

The time courses of the membrane potential of the SCN neuron model under different parameter values are shown in Figure 1. At default parameter values, the model showed spontaneous repetitive spiking (Figure 1(a)), as reported previously (Figure 2(a) in [2]). When only $g_{C a}$ was completely blocked, the model showed the stable steady state instead of the spontaneous spiking state (Figure 1(b)). When only $g_{N a}$ was completely blocked, the model showed a similar pattern, as illustrated in Figure 1(b) (Figure 1(c)). When both $g_{C a}$ and $g_{N a}$ were set to be values larger than the default values, the model showed the stable steady state (Figure 1(d)). However, the steady state membrane potential value shown in Figure 1(d) was more depolarized than that shown in Figure 1(b) and Figure 1(c).

Figure 2 illustrates the phase diagram of the model in the $\left(g_{C a}, g_{N a}\right)$-parameter space. The dynamical states of the model were classified into three states: the hyperpolarized steady state (white circle), the spontaneous spiking state (black circle), and the depolarized steady state (double circle). A decrease in $g_{N a}$ induced a decrease in the $g_{C a}$ range in which repetitive spiking occurred, and finally, the $g_{C a}$ range disappeared. Therefore, it is concluded that when $g_{N a}$ is zero, repetitive spiking cannot occur, no matter how large the $g_{C a}$ value is. An increase in $g_{N a}$ induced a decrease in the $g_{C a}$ threshold required to induce the transition from the hyperpolarized steady state to the spiking state. For example, even when $g_{C a}$ greatly decreased to $30 \mathrm{nS}$, repetitive spiking occurred under conditions in which $g_{N a}$ greatly increased to $1603 \mathrm{nS}$ (a seven-fold increase from the default value) (Figure 3(a)). In addition, even when $g_{C a}$ was zero, repetitive spiking occurred under conditions in which $g_{N a}$ was 1603 nS (Figure 3(b)).

\section{Discussion}

The present study revealed the sensitivity of the model dynamics to variations in $g_{C a}$ and $g_{N a}$ of circadian pacemaker neurons. Although Sim and Forger revealed the difference in time courses between the calcium and sodium currents (Figure 2(c) and Figure 2(d), respectively, in [2]), they did not clarify whether the calcium and sodium conductances are indispensable to repetitive spiking under the default condition. The present results (Figures 1(a)-(c)) resolved this issue; both $g_{C a}$ and $g_{N a}$ were indispensable to repetitive spiking under the default condition. Interestingly, the present study also revealed the difference between the two conductances. When we considered repetitive spiking under the condition in which the $g_{C a}$ value was smaller than its default value but the $g_{N a}$ value was much larger than its default value, the sodium conductance was indispensable but the 
(a) $g_{\mathrm{Ca}}=65 \mathrm{nS}, g_{\mathrm{Na}}=229 \mathrm{nS}$

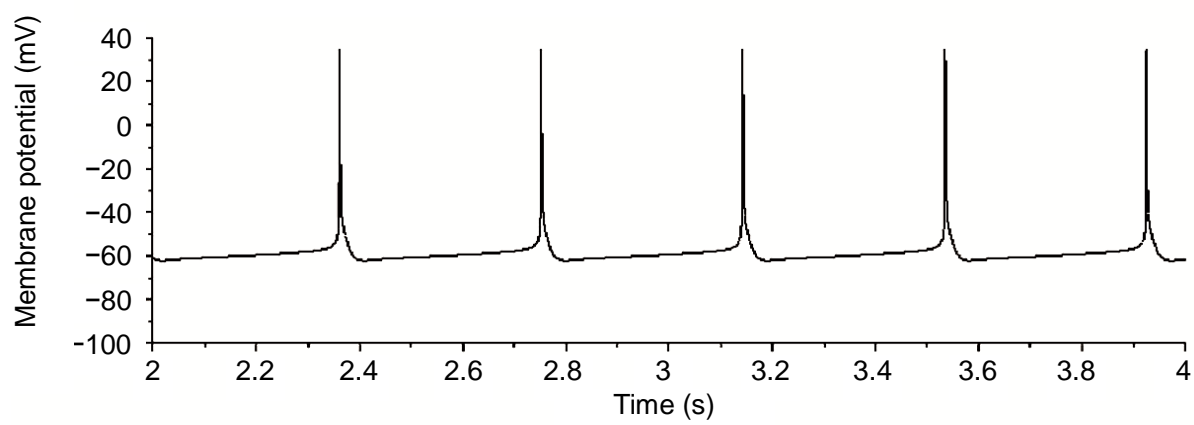

(b) $g_{\mathrm{Ca}}=0 \mathrm{nS}, g_{\mathrm{Na}}=229 \mathrm{nS}$

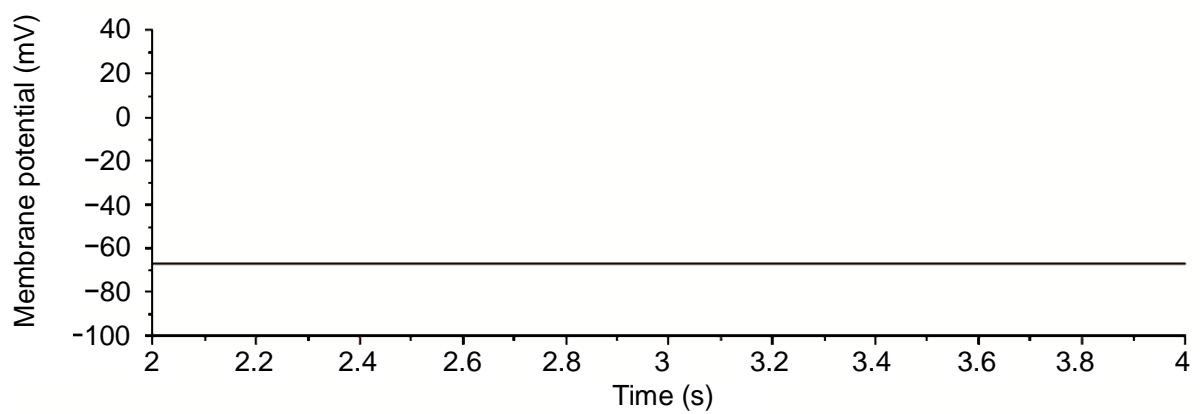

(c) $g_{C a}=65 \mathrm{nS}, g_{\mathrm{Na}}=0 \mathrm{nS}$

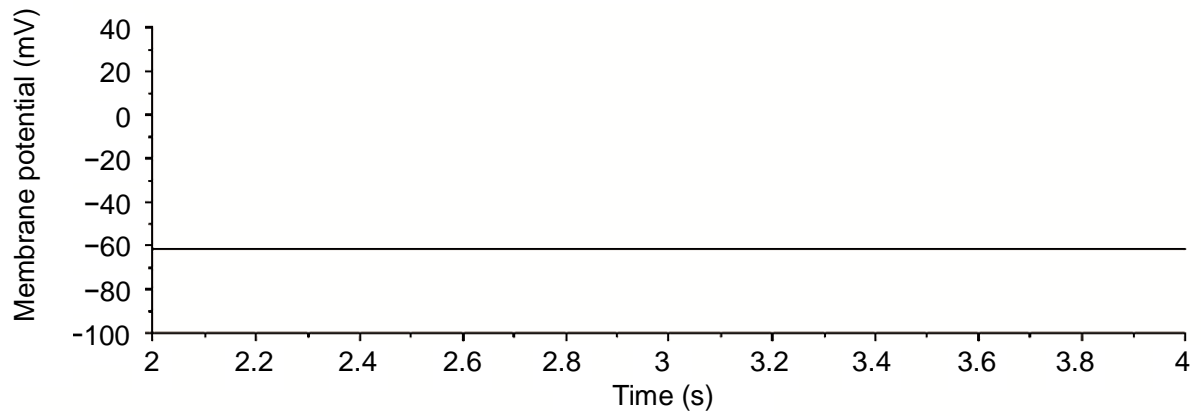

(d) $g_{\mathrm{Ca}}=80 \mathrm{nS}, g_{\mathrm{Na}}=350 \mathrm{nS}$

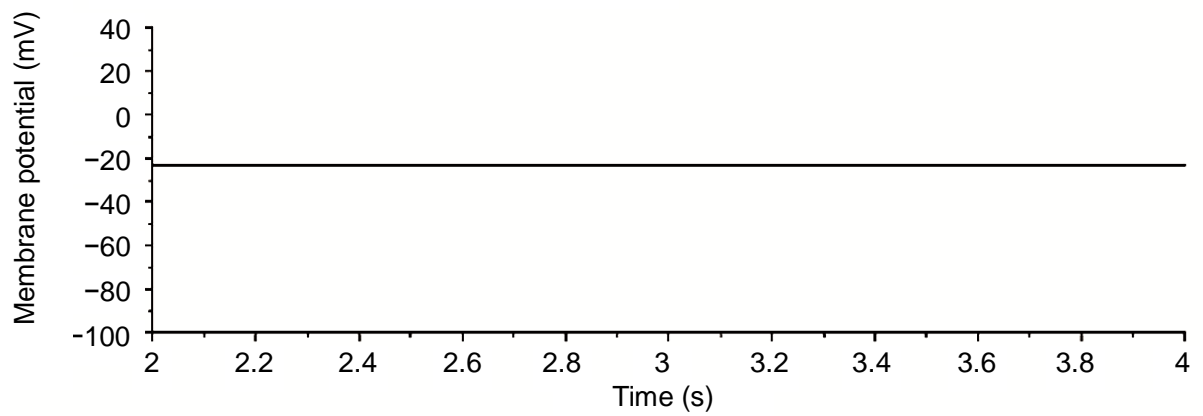

Figure 1. Time courses of the simulated membrane potential of circadian pacemaker neurons under several $\left(g_{\mathrm{Ca}}, g_{\mathrm{Na}}\right)$ conditions. (a) The spontaneous repetitive spiking state at $\left(g_{\mathrm{Ca}}, g_{\mathrm{Na}}\right)=$ $(65 \mathrm{nS}, 229 \mathrm{nS})$. (b) The hyperpolarized steady state at $\left(g_{\mathrm{Ca}}, g_{\mathrm{Na}}\right)=(0 \mathrm{nS}, 229 \mathrm{nS})$. (c) The hyperpolarized steady state at $\left(g_{C a}, g_{N a}\right)=(65 \mathrm{nS}, 0 \mathrm{nS})$. (d) The depolarized steady state at $\left(g_{\mathrm{Ca}}\right.$, $\left.g_{N a}\right)=(80 \mathrm{nS}, 350 \mathrm{nS})$. 
O: hyperpolarized steady state,

: spiking,

(O): depolarized steady state,

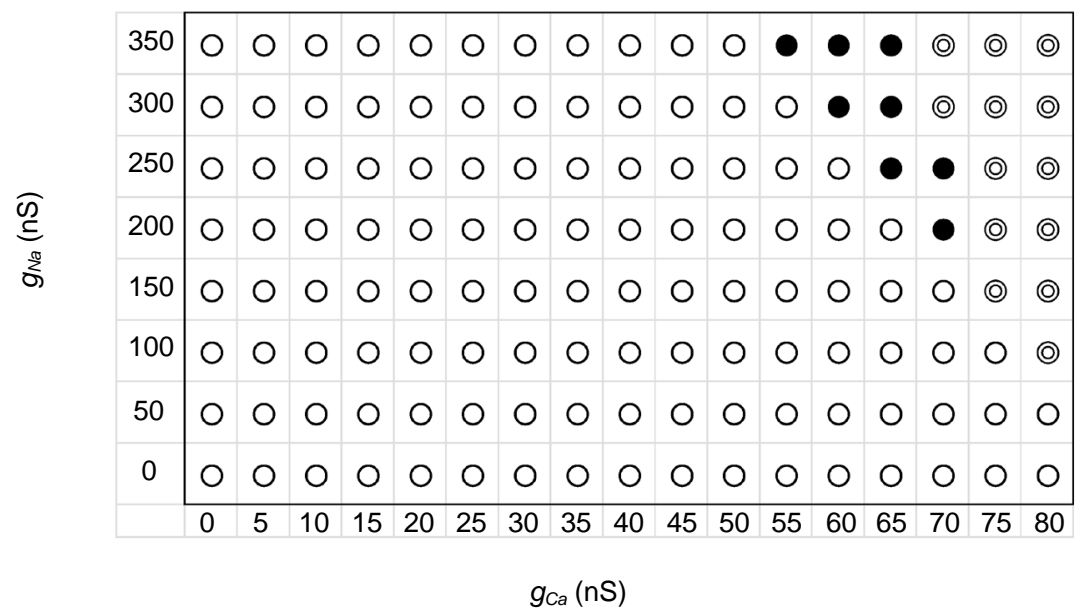

Figure 2. The sensitivity of the dynamical states of the circadian pacemaker neuron model to $g_{\mathrm{Ca}}$ and $g_{N a}$ variations. Black circle: the spontaneous repetitive spiking state, white circle: the hyperpolarized steady state, white double circle: the depolarized steady state.

(a) $g_{C a}=30 \mathrm{nS} \cdot g_{\mathrm{Na}}=1603 \mathrm{nS}$

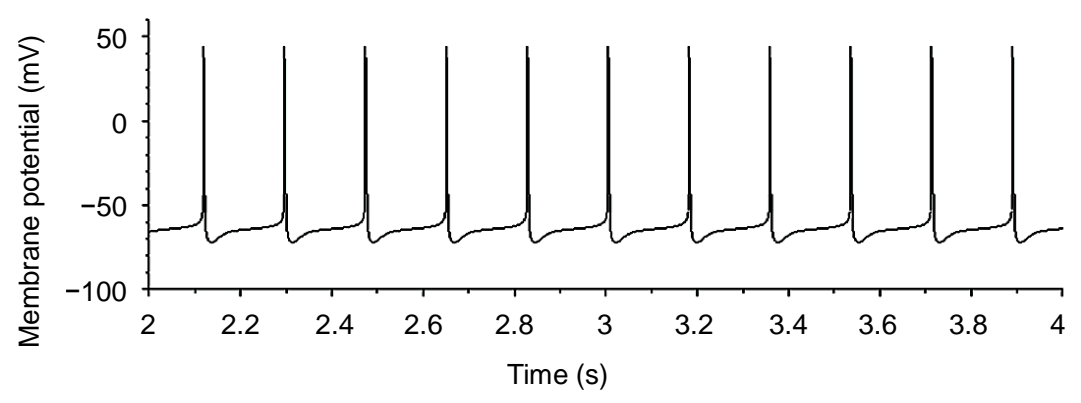

(b) $g_{\mathrm{Ca}}=0 \mathrm{nS} . g_{\mathrm{Na}}=1603 \mathrm{nS}$

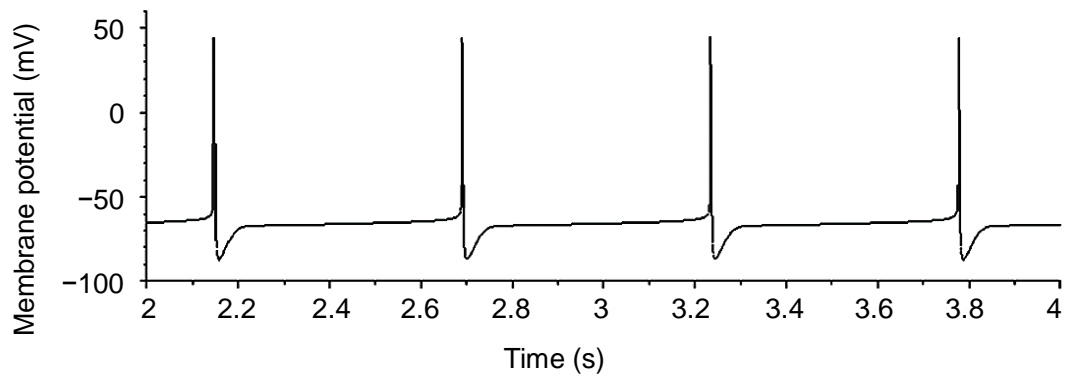

Figure 3. Time courses of the simulated membrane potential of circadian pacemaker neurons under $\left(g_{C a}, g_{N a}\right)$ conditions different from those in Figure 1. (a) The spontaneous repetitive spiking state at $\left(g_{\mathrm{Ca}}, g_{\mathrm{Na}}\right)=(30 \mathrm{nS}, 1603 \mathrm{nS})$. (b) The spontaneous repetitive spiking state at $\left(g_{\mathrm{Ca}}, g_{\mathrm{Na}}\right)=(0$ $\mathrm{nS}, 1603 \mathrm{nS})$. 
calcium conductance was dispensable to this type of repetitive spiking (Figure 2 and Figure 3(a) and Figure 3(a)).

Previous studies have reported the relationship between ionic conductances and neuronal spiking in various types of mathematical models [4]-[7]. In particular, similar to the circadian pacemaker neuron model analyzed in the present study, a mathematical model of midbrain dopaminergic neurons included the sodium and calcium conductances [4]. In the midbrain dopaminergic neuron model (Figure 4 in [4]), (1) an increase in $g_{N a}$ induced a decrease in the $g_{C a}$ threshold required to induce the transition from the nonpacemaking state to the pacemaking state and (2) the pacemaking occurred in the absence of the calcium conductance under conditions in which the sodium conductance was set to a large value. The present circadian pacemaker neuron model also exhibited these two characteristics. However, the present results revealed two important differences between these two models: (1) the $\left(g_{C a}, g_{N a}\right)$-parameter space was divided into only two states (the pacemaking and nonpacemaking states) in the midbrain dopaminergic neuron model (Figure 4 in [4]), whereas the ( $g_{C a}, g_{N a}$ )-parameter space was divided into three states (the depolarized steady state, the repetitive spiking state, and the hyperpolarized steady state) in the circadian pacemaker neuron model (Figure 2), and (2) pacemaking occurred in the absence of the sodium conductance under the condition in which the calcium conductance was set to a large value in the midbrain dopaminergic neuron model (Figure 4 in [4]), whereas pacemaking did not occur in the absence of the sodium conductance even when the calcium conductance was set to a large value in the circadian pacemaker neuron model (Figure 2).

\section{Conclusion}

The present investigation focused on a mathematical model of circadian pacemaker neurons, performed numerical simulation analysis, and compared this numerical result with that of the previous studies. The important and novel findings of the present study are as follows: 1) there was a difference in the contribution to repetitive spiking under certain conditions between the sodium and calcium conductances: for the generation of repetitive spiking under certain conditions, the sodium conductance played an indispensable role, whereas the calcium conductance was not necessarily essential, and 2) the $\left(g_{C a}, g_{N a}\right)$-parameter space of the circadian pacemaker neuron model showed a different pattern compared with that of the midbrain dopaminergic neuron model. These findings can contribute to our in-depth understanding of the influence of the sodium and calcium conductances on neuronal repetitive spiking.

\section{Acknowledgements}

The author would like to thank Enago (www.enago.jp) for the English language review.

\section{References}

[1] Ermentrout, G.B. and Terman, D. (2010) Mathematical Foundations of Neuroscience (Interdisciplinary Applied Mathematics). Springer, New York. http://dx.doi.org/10.1007/978-0-387-87708-2

[2] Sim, C.K. and Forger, D.B. (2007) Modeling the Electrophysiology of Suprachiasmatic Nucleus Neurons. Journal of Biological Rhythms, 22, 445-453. http://dx.doi.org/10.1177/0748730407306041

[3] Ashrafuzzaman, M. and Tuszynski, J. (2012) Membrane Biophysics (Biological and Medical Physics, Biomedical Engineering). Springer, New York.

[4] Drion, G., Massotte, L., Sepulchre, R. and Seutin, V. (2011) How Modeling Can Reconcile Apparently Discrepant Experimental Results: The Case of Pacemaking in Dopaminergic Neurons. PLoS Computational Biology, 7, e1002050. http://dx.doi.org/10.1371/journal.pcbi.1002050

[5] Shirahata, T. (2011) The Effect of Variations in Sodium Conductances on Pacemaking in a Dopaminergic Retinal Neuron Model. Acta Biologica Hungarica, 62, 211-214. http://dx.doi.org/10.1556/ABiol.62.2011.2.11

[6] Shirahata, T. (2014) Effect of Sodium Conductance Variations on Electrical Behavior of a Neocortical Neuron Model. Acta Biologica Hungarica, 65, 379-384. http://dx.doi.org/10.1556/ABiol.65.2014.4.2

[7] Shirahata, T. (2015) Numerical Study of a Mathematical Model of Vibrissa Motoneurons: The Relationship between Repetitive Spiking and Two Types of Sodium Conductance. International Journal of Theoretical and Mathematical Physics, 5, 48-52. 